\title{
AN INFINITESIMAL RATTRAY THEOREM
}

Let $X$ be a continuous vector field on the unit Euclidean sphere $S^{n-1} \subset \mathbb{R}^{n}$ centered at the origin. It is proved that if $X(-a)=-X(a)$ holds true for each vector $a$, then there is an orthonormal basis such that for any two vectors a and $b$ in the basis one has $X(a) \cdot b+a \cdot X(b)=0$. Bibliography: 1 title.

It is well known that if two unit orthogonal vectors $a$ aud $b$ in $\mathbb{R}^{n}$ smoothly depend on time, then we have the relation

$$
a^{\prime} \cdot b+a \cdot b^{\prime}=0
$$

Theorem. Let $X$ be a continuous vector field on the unit Euclidean sphere $S^{n-1}$ in $\mathbb{R}^{n}$ centered at the origin. Assume that we have $X(-a)=-X(a)$. Then there is an orthonormal basis in $\mathbb{R}^{n}$ such that for any two vectors $a$ and $b$ in the basis we have $X(a) \cdot b+a \cdot X(b)=0$.

Proof. Shift along trajectories of the vector field $X$ determines an antipodal continuous mapping of the sphere: $S^{n-1} \rightarrow S^{n-1}$. By the Rattray Theorem [1], the mapping maps a certain orthonormal basis to another orthonormal basis. Since for small times $t$ the shift of a point $a$ of the sphere is approximately equal to $X(a) t$, it follows that passing to the limit as $t \rightarrow 0$ we obtain the required assertion.

Remark. We have actually proved a theorem about tangent vector fields on the projective space because the considered vector fields $X$ on the sphere $S^{n-1}$ induce vector fields on the projective space, and vice versa.

Anti-symmetrizing an arbitrary vector field on the sphere, we obtain the following corollary.

Corollary. Let $X$ be a continuous vector field $X$ on $S^{n-1}$. Then there is an orthonormal basis in $\mathbb{R}^{n}$ such that for any two basis vectors $a$ and $b$ we have the relation

$$
(X(a)-X(-a)) \cdot b+a \cdot(X(b)-X(-b))=0
$$

Translated by V. V. Makeev.

\section{REFERENCES}

1. R. Rattray, "An antipodal point, orthogonal point theorem," Ann. Math., 80, 502-512 (1954).

\footnotetext{
${ }^{*}$ St. Petersburg State University, St.Petersburg, Russia, e-mail: mvv57@inbox.ru.
}

Translated from Zapiski Nauchnykh Seminarov POMI, Vol. 353, 2008, pp. 148-149. Original article submitted July 21, 2006 .

436 